\title{
LA PRÁCTICA EDUCATIVA BAJO EL LENTE DE LA TEORÍA DE LA ACTIVIDAD
}

\author{
Ariana De Vincenzi ${ }^{1^{*}}$, Dairaliz Marcano ${ }^{2}$ y Alejandra Macri ${ }^{3}$ \\ 1,2,3 Universidad Abierta Interamericana, Buenos Aires, Argentina. \\ *Autor de correspondencia: adevincenzi@uai.edu.ar
}

Recibido Octubre 2020; Aceptado Diciembre 2020

\begin{abstract}
Resumen - El artículo aborda el proceso de construcción del método propuesto para analizar buenas prácticas educativas como sistemas de actividad, considerando su configuración, contradicciones, historicidad y multivocalidad, en el marco de una investigación que estudia la calidad educativa como transformación en una universidad privada argentina. Dos preguntas guiaron el proceso de investigación documental: cómo identificar y seleccionar las buenas prácticas objeto de análisis y bajo qué método investigarlas. Se consideraron treinta y ocho investigaciones que emplearon la metodología de la teoría de la actividad para analizar prácticas educativas en contextos educativos; ocho se analizaron en profundidad para identificar las categorías e indicadores de la práctica educativa como sistema de actividad. La información recogida en matrices sirvió de base para la construcción del método que integra la teoría de la actividad y las dimensiones didácticas para el análisis de las buenas prácticas. Se concluye que las categorías y preguntas del método elaborado abonan a la construcción de esquemas de investigación para el estudio en profundidad de las buenas prácticas en el marco de la calidad con foco en las transformaciones formativas.
\end{abstract}

Palabras clave: calidad educativa, teoría de la actividad, universidad, buenas prácticas, sistema de actividad.

\section{EDUCATIONAL PRACTICE THROUGH THE LENS OF THEORY OF ACTIVITY}

\begin{abstract}
The article addresses the process of construction of the proposed method to analyze good educational practices as activity systems, considering their configuration, contradictions, historicity and multivocality, within the framework of a research that studies educational quality as transformation in an Argentine private university. Two questions guided the documentary research process: how to identify and select the good practices to be analyzed and under what method to investigate them. Thirty-eight investigations were considered that used the methodology of activity theory to analyze educational practices in classroom contexts; eight were analyzed in depth to identify the categories and indicators of educational practice as an activity system. The information collected in matrices served as the basis for the construction of the method that integrates the theory of the activity and the didactic dimensions for the analysis of good practices. It is concluded that the categories and questions of the elaborated method contribute to the construction of research schemes for the in-depth study of good practices in the framework of quality with a focus on formative transformations.
\end{abstract}

Keywords: educational quality, theory of activity, university, good practices, activite system. 


\section{Introducción}

El estudio de la práctica educativa universitaria como práctica social, contextualizada y plurideterminada, supone la adopción de metodologías que visibilicen la trama de múltiples determinaciones de orden intra e extra didáctico que estructura y dinamiza la interacción docente, estudiantes y contexto. La investigación del aula universitaria como microespacio que articula lo subjetivo y lo social (Lucarelli, 2006) requiere de lentes que permitan captar la acción humana que se sucede en su interior y "que amarra a los actores por individualizables que sean, a una historia social compartida” (Edelstein, 2000, p. 5).

En el marco de la calidad educativa a nivel universitario con foco en las transformaciones formativas (Harvey y Green, 1993), se planteó una investigación con el objetivo de analizar la buena práctica educativa concebida como un espacio de intercambios de significados socioculturales entre los estudiantes y el docente, en un contexto y temporalidad singular y con base en determinados propósitos (Gimeno y Pérez, 1992; Zabalza, 2012). Constituyen intervenciones educativas que facilitan el desarrollo de actividades de aprendizaje en las que se logran con eficiencia los resultados formativos previstos y también otros aprendizajes de valor educativo (Grupo DIM-UAB, 2014).

Con base en la definición de buena práctica educativa asumida como punto de partida, la enseñanza se aborda dentro de la perspectiva ecológica, con acento en el rol activo del sujeto en su aprendizaje y en la mediación cultural del docente en la enseñanza. Supone una ruptura de la díada estructural docente enseña y estudiante aprende, dando paso a la idea de la co-creación de ambientes de aprendizaje donde docente y estudiantes accionan de forma combinada e interrelacionada. La formación surge de esfuerzos colaborativos dirigidos al logro de metas compartidas, con participación de distintas perspectivas a través del diálogo y del cuestionamiento. Dicha formación se encuadra en un contexto que le otorga significación y al cual ésta, a su vez, modifica.

Tres conceptos marcaron teóricamente el estudio de la enseñanza dentro de la investigación. En primer término, la noción de cognición distribuida (Salomon, 1993; Perkins, 2001), el conocimiento se construye en el contexto de personas que piensan en conjunto con ayuda de herramientas y medios proporcionados por la cultura. La conformación de comunidades de aprendizaje (Wenger, 2010) que comparten un mismo interés y convergen en un sistema de actividad. En tercer lugar, la noción de la enseñanza como proceso que excede los límites del aula, articulando saberes y experiencias de aprendizaje que tienen sentido al conectarse con estructuras tanto dentro como fuera de la institución educativa.

Para el estudio de la buena práctica educativa como unidad de análisis, Zabalza (2012) identifica algunas características epistemológicas. En primer lugar, la buena práctica educativa es un conjunto de acciones intencionales que surge en un contexto que la condiciona, por lo que su análisis en el nivel universitario debe contemplar aspectos del contexto institucional, su organización y dinámica, la disciplina, el currículum, las exigencias formativas del perfil de egreso, entre otros. La segunda característica hace referencia a la bidireccionalidad de la acción educativa como acto personal de docentes y estudiantes, y a la vez, como realidad cultural que dota de significados, donde el análisis de una buena práctica es más complejo que ver lo que se hace, debe penetrar motivaciones y sentido de las acciones.

IPSA SCIENTIA - Vol. 5 Nº $1-2020$ 
El análisis de una buena práctica debe dar cuenta de las acciones desarrolladas en elproceso de enseñanza, como tercera característica. Por último y cuarto término, una buena práctica lo es no sólo por los elementos técnicos o los resultados.

En la investigación educativa, la teoría de la actividad (Engeström, 2001, 2014) constituye un marco metodológico para el estudio de interacciones educativas dentro y fuera del aula en consonancia con los marcos conceptuales de la enseñanza y las características epistemológicas de las buenas prácticas antes descritos. La práctica educativa como sistema de actividad es entendida como acción humana intencional que convoca la participación de estudiantes y profesores en torno a un objeto común que los motiva y dirige, haciendo uso de herramientas físicas y socio culturales que facilitan el logro de los resultados esperados. Analiza la cultura del aula de clases desde distintas capas, mediada por herramientas en constante desarrollo histórico y desde diferentes perspectivas (Arnseth, 2008; Huang, 2011; Juárez, Buenfil y Triguelos, 2008; Mattar, 2018).

Estudiar la enseñanza desde este enfoque, supone analizar la complejidad del proceso formativo en su propio contexto para acceder a la acción pedagógica y dispositivos que actúan en una zona de desarrollo próximo (Vygostky, 1978), complejizando los mecanismos, factores y sistemas sociales y culturales que dan sentido a lo que sucede en el aula de clase.

El sistema de actividad educativo está compuesto de acciones interrelacionadas de alumnos, docentes y autoridades, inmersos en un contexto político y social, culturalmente organizado y mediados por herramientas, donde todos se comprometen, promulgan y trabajan por un objeto que motiva y da dirección a la actividad, y que, a su vez, es transformado por sus acciones (Christiansen,1996). La actividad generada en el aula de clase muestra una dinámica de intercambio con identidad propia, que refleja los intercambios sociohistóricos y culturales que sitúan su desarrollo (Hardman, 2008). Su abordaje amerita enfoques capaces de captar la complejidad, brindando explicaciones y significados de actores enculturados por los valores y recursos de su cultura, a la luz de las trayectorias históricas que configuran sus acciones (Sannino y Engeström, 2018).

El presente artículo describe el método propuesto para analizar la práctica educativa como sistema de actividad, considerando su configuración, contradicciones, historicidad y multivocalidad (Engeström, 2014) en el marco de una investigación que estudia la calidad educativa como transformación en una universidad privada argentina.

En tal sentido, dos preguntas guiaron la aproximación a las buenas prácticas como objeto de investigación: cómo identificar y seleccionar las experiencias a analizar y bajo qué método investigarlas.

\section{El sistema de actividad educativo como unidad de análisis}

La actividad humana constituye la unidad de análisis en el marco de la teoría histórico-cultural definida como las acciones (actuaciones) intencionales de personas que operan en forma conjunta sobre un objeto que las motiva y dirige, haciendo uso de herramientas que facilitan el logro de resultados (Vygostky, 1978; Leontiev, 1981; Engeström, 1987). 
La actividad se configura en un sistema, una estructura de relaciones, que admite el análisis de las acciones en un marco que tiene en cuenta lo que las personas hacen juntas en cuanto colectivos que colaboran para el logro del objeto. En este sentido, para Leontiev (1981), la actividadconstituye la unidad básica no reductible a unidades discretas de acción individual; es decir, el logro del objeto tiene cabida únicamente a partir de la actuación conjunta de las personas que participan en el sistema y no por la acción particular de los individuos.

El análisis de aquello que la gente hace, si bien implica su abordaje desde la acción humana en marcha, lleva la impronta de la cultura y la historia del contexto donde la actividad tiene lugar. La cultura modela las acciones de los sujetos de la actividad con base a los valores, a la provisión y modos de uso de herramientas de mediación (materiales y simbólicas) y dota al objeto de relevancia.

Las trayectorias históricas marcan las acciones en tres direcciones. Por un lado, las acciones de las personas adquieren significado situadas en el contexto temporal donde tienen lugar y considerando su evolución en el tiempo. Otra parte, las herramientas que median las acciones de los sujetos hacia el logro del objeto llevan consigo rastros del proceso evolutivo que les ha dado el papel de mediación. Por último, el objeto de la actividad evoluciona y se transforma al paso de las acciones y de las herramientas de mediación (Daniels, 2016).

El carácter histórico y cultural del sistema de actividad tiene su fundamento en el supuesto de que la actividad humana y el aprendizaje consciente son completamente interactivos e interdependientes (Leontiev, 1981). Ninguna actividad puede ocurrir sin la intervención de los procesos de pensamiento consciente, $\mathrm{y}$, al mismo tiempo, ningún pensamiento consciente puede ocurrir fuera del contexto de una actividad. Por lo tanto, el análisis del sistema de actividad da cuenta tanto de los aprendizajes que surgen en el curso de la actividad como de los adquiridos por los participantes.

Un sistema de actividad, con base en los postulados teóricos de Vygostky (1978) y los aportes de Leontiev (1981) al incorporar la dimensión colectiva, se define como cualquier interacción humana en curso, dirigida a objetos, estructurada históricamente y mediada por herramientas. Es una categoría conceptual que puede explicar los sistemas de actividad que suceden en una familia, una organización laboral, un movimiento político, un curso de estudio, una escuela, una disciplina, entre otros (Russell, 2004).

Engeström (1987) afirma que, además de los elementos configurativos descritos por Vygotsky y Leontiev, es necesario considerar que los sujetos de la actividad actúan incorporados a una comunidad que comparte el interés por el logro del objeto e impone reglas y roles (división del trabajo) al sistema. De esta manera, el modelo original conocido como primera generación de TA se amplía y da cabida a la comunidad de interés, las reglas y la división del trabajo, en lo que se ha denominado la segunda generación del modelo (Figura 1).

Con la incorporación de la comunidad en el análisis, el sistema de actividad se organiza en múltiples voces y capas donde cada participante lleva su propia historia, puntos de vista, tradiciones e interés, artefactos de mediación, reglas, convenciones y roles. 
Figura 1. Configuración del sistema de actividad. Segunda generación

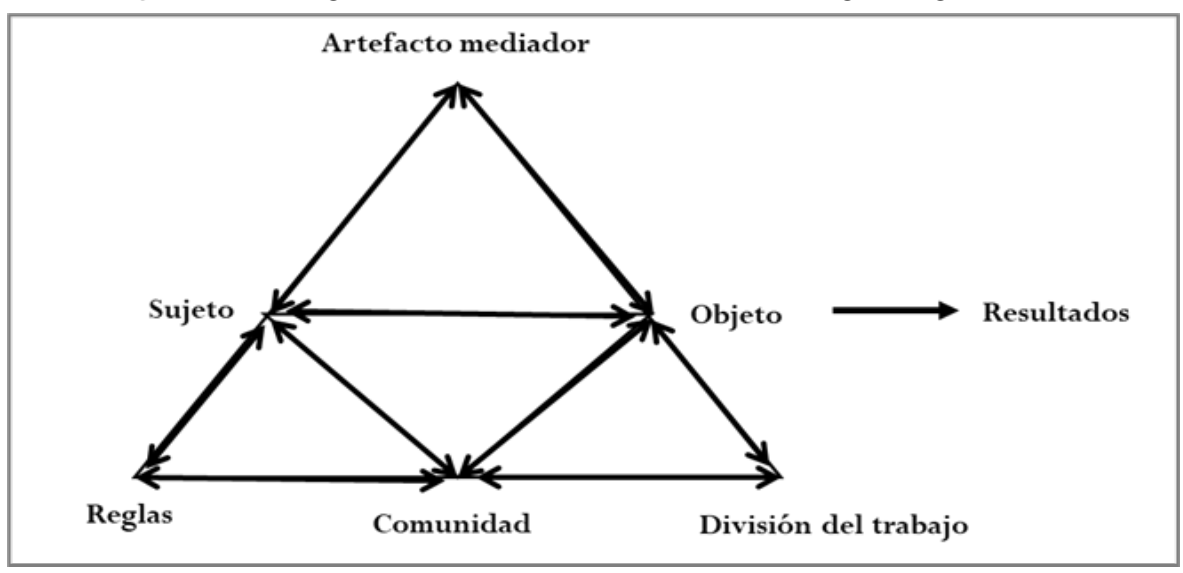

Fuente: Engeström (1987)

Para la teoría de la actividad las contradicciones, conflictos y rupturas son inevitables en el funcionamiento de cualquier sistema. Se explican bien como desajustes entre elementos de un sistema de actividad, como producto de la influencia entre sistemas de actividades que interactúan, o como resultado de perturbaciones producidas por influencias externas al sistema (Foot, 2014).

En cualquier caso, son el resultado de las tensiones estructurales históricamente acumuladas dentro y fuera del sistema de actividad, y representan oportunidades para innovaciones creativas, nuevas formas de estructuración y manifestación de la actividad. Engeström describe las contradicciones como bisagras iluminativas que pueden abrir nuevas perspectivas de comprensión al vincular la historia del sistema de actividad con su zona de desarrollo próximo, activando la posibilidad de transformaciones expansivas que se mueven en forma de ciclos de cambios cualitativos (Engeström, 2001, 2014).

Las investigaciones concluyen que la teoría de la actividad constituye un marco metodológico capaz de captar la complejidad de los sistemas de actividad educativa al organizar, tamizar, clasificar y aclarar complejos fenómenos dentro y fuera del aula (Kumpulainen y Sefton-Green, 2014). Como metodología, tiende un puente entre la teoría y la recolección y análisis de datos. Proporciona flexibilidad desde la perspectiva contextual con base en la distinción entre el sistema de actividad colectiva y la acción individual, en consonancia con el enfoque ecológico de la enseñanza, y da cabida a una variedad de enfoques e instrumentos para la investigación de prácticas educativas (Nussbaumer, 2012). Por las razones antes expuestas, se seleccionó como el marco metodológico para el análisis de buenas prácticas educativas en una investigación sobre calidad de transformación a nivel universitario. Seguidamente se analizan los aspectos a tener en cuenta para investigar buenas prácticas educativas.

\section{La investigación de buenas prácticas educativas.}

Para Zabalza (2012), los principales problemas que plantea la investigación de las buenas prácticas son: el establecimiento de criterios confiables para la identificación y selección de la práctica a analizar; la determinación de las dimensiones para deconstruir la realidad y la forma de acceder, de manera directa o indirecta, a la práctica. 
El mismo autor propone algunos criterios básicos a tomar en consideración, sea individualmente o en forma combinada, para identificar y seleccionar buenas prácticas: innovación (originalidad con respecto a las formas convencionales de actuación); mejora de recursos, espacios, modos de relación, etc.; actuaciones con fundamentación científica relacionadas con el aprendizaje; acciones didácticas o estrategias de aprendizaje con eficacia demostrada en su campo disciplinar; resultado institucional de evaluaciones docentes; alto grado de satisfacción de los estudiantes con la práctica educativa, responsables educativos y/u otros agentes implicados; impacto social referido al compromiso de las acciones educativas. Puntualiza que:

Las buenas prácticas incluyen, necesariamente, el objetivo de buenos aprendizajes (en calidad y cantidad) pero transciende esa zona restrictiva de lo instructivo para proponerse mejoras tanto en los ambientes de aprendizaje como en el valor y sentido de las cosas que se aprenden y en la forma en que se aprenden (p. 30).

En relación con la investigación de las prácticas de enseñanza, Edelstein (2000) focaliza su objetivo en el logro de un mayor nivel de comprensión posible, a partir de "la reflexión como reconstrucción crítica de la experiencia”, lo que demanda el contraste intersubjetivo y plural, dialógico y de debate, en respuesta a "un objeto de estudio que se desarrolla en contextos sociohistóricos concretos y no en ámbitos prefigurados” (p. 5). En tal sentido, la propuesta metodológica para la investigación de las prácticas educativas deberá incluir herramientas e instrumentos que evidencien su naturaleza y permitan avanzar desde la descripción hacia el análisis y la interpretación con base en las categorías teóricas.

Como lo menciona Zabalza (2012), para investigar buenas prácticas educativas es necesario determinar con precisión las categorías de análisis que den cuenta de sus características. A los fines de la investigación, se asumieron las seis dimensiones propuestas por Gimeno y Pérez (1989), desarrollada por una de las investigadoras en un estudio previo (De Vincenzi, 2009); a saber: planificación, estructuración metodológica del contenido, interrelaciones entre docente y estudiantes, evaluación, organización de la vida en el aula y el tipo de tareas académicas.

Para acceder a la práctica desde una perspectiva ecológica fue necesario diseñar el método que permitiera su análisis, considerando las dimensiones didácticas antes mencionadas, desde la configuración de la enseñanza como sistema de actividad (sujeto, herramientas, objeto, normas, comunidad, división de tarea y resultados), y los principios de historicidad, múltiples voces y contradicciones El presente artículo reporta el proceso de construcción del método utilizado para analizar las buenas prácticas educativas, integrando las dimensiones del análisis didáctico en el marco de la enseñanza como actividad ecológica compleja.

\section{Materiales y Métodos}

Se propuso una investigación de corte documental y analítica para la elaboración de un método que permitiera el estudio de la práctica educativa como sistema de actividad, atendiendo a la perspectiva del modelo ecológico de la enseñanza.

Eli de Gortari (1981, citado por Tena y Rivas - Torres, 1995) define el método como: 
El procedimiento planeado que se sigue en cada actividad científica para descubrir la forma de existencia de los procesos, distinguir las fases de su desarrollo, desentrañar sus enlaces internos y externos, esclarecer sus interacciones con otros procesos, generalizar y profundizar los conocimientos adquiridos de este modo, demostrarlos luego con rigor racional y conseguir después su comprobación en el experimento y con la técnica de su aplicación (p.17).

En este sentido, el método resultante debía incluir tanto las categorías que configuran el sistema de actividad (Engeström, 1987) como las dimensiones didácticas (Gimeno y Pérez, 1989) para analizar la forma en la cual las prácticas se constituyen en "buenas prácticas educativas”.

Se analizaron treinta ocho trabajos de investigación, en su mayoría en idioma inglés, que emplearon la metodología de la teoría de la actividad para identificar las categorías de configuración del sistema, historicidad, multivocalidad y contradicciones en prácticas educativas. Del primer muestreo, doce investigaciones emplearon la configuración del sistema de actividad y el principio de contradicción para evaluar la eficacia de estrategias de enseñanza en aulas de nivel primario, cuatro en educación secundaria y seis en enseñanza universitaria; tres analizaron la formación docente considerando además los conceptos de historicidad y múltiples voces; ocho configuraron situaciones de aprendizaje en contextos educativos no formales; nueve analizaron la incorporación de herramientas de mediación tecnológica en la enseñanza.

De la muestra inicial, se seleccionaron ocho investigaciones que definen indicadores para la observación de prácticas educativas como sistema de actividad, organizando matrices para cada componente del sistema. De la muestra analizada, no se encontraron indicadores que contemplaran las categorías didácticas. Por tal motivo, se decidió construir un método que integrara ambos marcos metodológicos.

Las investigaciones analizadas no reportaron parámetros para identificar y seleccionar buenas prácticas. Para dar respuesta al objetivo, se seleccionaron dos de los criterios propuestos por Zabalza (2012): resultados institucionales de la evaluación docente y prácticas con alto grado de satisfacción por parte de responsables educativos y estudiantes. La selección de las prácticas educativas se hizo atendiendo al juicio experto de la unidad de gestión pedagógica de la universidad a cargo de las evaluaciones de seguimiento a los docentes y a la recomendación de los decanos de las facultades.

El instrumento utilizado fue el modelo de configuración del sistema de actividad en su segunda generación (Figura 1), ubicando en el vértice superior del gráfico (artefacto de mediación) las categorías de planificación, estructuración metodológica del contenido, interrelaciones entre docente y estudiantes, evaluación, organización de la vida en el aula y el tipo de tareas académicas. Se elaboraron preguntas atendiendo a las categorías de sujeto, objeto, comunidad, reglas y división del trabajo, en sesiones de trabajo a cargo de las investigadoras, contrastando con la matriz resultante de la revisión de las investigaciones. 


\section{Resultados y Discusión}

\section{Identificación y selección de las buenas prácticas}

La investigación de las buenas prácticas educativas con foco en el sistema de actividad se realiza en una universidad privada argentina que, en su proyecto institucional, explicita su orientación pedagógica hacia la formación de profesionales en contextos colaborativos de trabajo, articulados con el medio externo. Desde su creación, la universidad cuenta con una unidad de gestión pedagógica que asesora a los profesores mediante observaciones de clases, programas de capacitación pedagógica, encuentros de reflexión sobre sus prácticas educativas e informes anuales de desempeño docente. Estos informes de evaluación se triangulan con los resultados de las fichas de opinión sobre la calidad de la docencia que los alumnos completan al cierre de cada semestre y con los resultados de las fichas de evaluación docente que elaboran los directivos de las carreras.

Atendiendo a los criterios para la identificación y selección de las buenas prácticas educativas (Zabalza, 2012) se consideraron los resultados institucionales de la evaluación docente y la satisfacción de los implicados (autoridades decanales, estudiantes y asesores pedagógicos) en cuanto a que en ambas se reconocen la originalidad y eficiencia de las estrategias de enseñanza aprendizaje y los resultados de aprendizaje alcanzados.

Con base a lo anterior, se definieron los dos primeros criterios para la selección de las buenas prácticas objeto de estudio: a) Trayectorias de prácticas docentes que evidencien valoraciones positivas en fichas de observaciones de clases, fichas de opinión de estudiantes y fichas de evaluación de los directivos. b) Docentes de carreras incorporadas en el art. $43^{\circ}$ de la Ley de Educación Superior 24.521, que hayan participado al menos de dos acreditaciones por la Comisión Nacional de Evaluación y Acreditación Universitaria (CONEAU) ${ }^{1}$. El tercer criterio se estableció conforme la concepción ecológica de la enseñanza: prácticas que promovieran el trabajo colaborativo en comunidades de aprendizaje en torno a un interés común.

Como resultado del proceso, se seleccionaron cuatro buenas prácticas, dos pertenecientes a la carrera de Arquitectura y dos a la carrera de Psicología. Para el momento que se escribe el presente artículo, la investigación se encuentra en fase de análisis de resultados.

\section{Categorías de análisis propuestas por las investigaciones de la práctica educativa como sistema de actividad}

Del análisis documental de las investigaciones que proponen las categorías del sistema de actividad para analizar la práctica educativa en el aula, se construyeron las definiciones y matrices de categorías observadas. Seguidamente, se presentan los resultados de este primer

\footnotetext{
${ }^{1}$ La Comisión Nacional de Evaluación y Acreditación Universitaria (CONEAU) es un organismo descentralizado que funciona en jurisdicción del Ministerio de Educación de la Nación Argentina. Su misión institucional es asegurar y mejorar la calidad de las carreras e instituciones universitarias que operan en el sistema universitario argentino por medio de actividades de evaluación y acreditación de la calidad de la educación universitaria. (CONEAU, 2020)
} 
proceso de construcción de categorías, que más tarde sería fundamental para la elaboración y triangulación de las categorías definitivas utilizadas para la investigación en curso.

Los sujetos de la actividad: Docentes y estudiantes se ubican en uno de los vértices en relación con el objeto y mediados por herramientas. Llegan al sistema de actividad en curso con intencionalidades, suposiciones acerca del aprendizaje y sus resultados, la enseñanza, la historia de herramientas de mediación sociocultural presentes y pasadas (Russell, 2004; Salas-Madrid, 2016).

Las herramientas de mediación: Son los dispositivos físicos (aparatos y herramientas físicas) o simbólicas (lenguaje, modelos, habilidades, actitudes), incorporados en un sistema de actividad y que cumplen una función de mediación entre el sujeto y los objetos (Hardman, 2008; Russell, 2004). En el caso de la práctica educativa, la didáctica organiza los dispositivos mediacionales entre los estudiantes y el objeto de aprendizaje. Las categorías de planificación, estructuración metodológica del contenido, interrelaciones entre docente y estudiantes, evaluación, organización de la vida en el aula y el tipo de tareas académicas constituyen artefactos culturales implicados que desempeñan un papel fundamental para dar forma a la acción educativa utilizada por docentes y estudiantes (ver tabla 1).

Tabla 1: Herramientas de mediación

\begin{tabular}{ll}
\hline \multicolumn{1}{c}{ Categoría } & \multicolumn{1}{c}{ Indicadores } \\
\hline \multirow{2}{*}{ Materiales y recursos } & Pizarra. \\
& Recursos tecnológicos \\
\hline & Preguntas/respuestas del docente \\
& Preguntas/respuestas del alumno \\
& Consultas de fuentes de datos primario (campo) \\
Comunicativas & Lectura. Recursos de audio y video \\
& Expresiones orales y escritas del docente y del alumno \\
& Ejemplos. Metáforas. Gráficos. Casos y ejercicios de estudio. Algoritmo. \\
& Modelos \\
\hline \multirow{3}{*}{ Didáctica } & Objetivos/metas \\
& Estructuración de los contenidos \\
& Estrategias de enseñanza (deductivas, cooperativas, enseñanza directa, indagación) \\
& Estrategias de evaluación \\
\hline
\end{tabular}

Fuente: Elaboración propia

El objeto: El objeto de estudio convoca a los sujetos, motiva y dirige la práctica y remite a la intencionalidad educativa. Debe ser atractivo para los estudiantes y el docente. Todo objeto comporta una ambigüedad intrínseca: es portador de un significado cultural y es significado por los sujetos (Jonassen, 2000). Como lo afirma Solórzano y García (2016), docentes y estudiantes, construyen en interrelación colaborativa "entramados semánticos acordes a las reflexiones desarrolladas por el grupo” (p.105), plasmados en elementos culturales (escritos, orales, materiales) que dan sentido a la acción.

Las reglas: Constituyen las pautas y normas que regulan, organizan y dirigen las relaciones entre los sujetos y entre estos con el objeto, con las herramientas empleadas y sus formas de utilización y con la comunidad con la que comparten el mismo interés por el objeto (Foot, 2014). Hardman (2008) categoriza las reglas de los sistemas de actividad pedagógica en institucionales, instruccionales y de interacción (ver tabla 2). 
La práctica educativa bajo el lente de la teoría de la actividad

Tabla 2: Reglas

\begin{tabular}{cl}
\hline Categoría & \\
\hline Institucionales & Proyecto académico \\
& Reglamentos \\
& Política institucional \\
\hline Instruccionales & Criterios de evaluación \\
& Consignas \\
\hline \multirow{2}{*}{ De interacción } & De orden y disciplina \\
& Distribución de tiempo y recursos \\
\cline { 2 - 2 } & Expresión comunicativa y afectiva \\
\hline
\end{tabular}

Fuente: Hardman (2008)

Comunidad: Docentes y estudiantes en un aula conforman una comunidad educativa con características singulares que condicionan los resultados del proceso formativo. En tanto que constituyen una comunidad de práctica, convergen en un sistema de actividad donde comparten un interés común (objeto), con arreglo a determinadas herramientas y organizados en torno a roles (Wegner, 2010). El aprendizaje ocurre más allá del individuo; es en la comunidad, en tanto espacio de diálogo integrado por docentes, estudiantes y otros actores institucionales, donde este se construye (Solórzano y García, 2016). Se muestran en la tabla 3.

Tabla 3: Comunidad

\begin{tabular}{ll}
\hline Microcultura académica & Pertenencia a grupos de trabajo académico \\
\hline Organización del grupo-clase & Grupos permanentes de estudiantes \\
Grupos transitorios de estudiantes
\end{tabular}

Fuente: Elaboración propia

División del trabajo: En un plano horizontal, establece qué miembros de la comunidad participan en delimitados tipos de acciones y qué uso hacen de determinadas herramientas; mientras que el grado de control y acceso a recursos y recompensas configura el plano vertical (Ventak y Adler, 2008; Salas-Madrid, 2016). Resultado del análisis documental se discriminaron las categorías mostradas en la tabla 4.

Tabla 4: División del trabajo

\begin{tabular}{|c|c|c|}
\hline & Categoría & Indicador \\
\hline \multirow[t]{3}{*}{ Rol del docente } & Instructor & $\begin{array}{l}\text { Presenta los contenidos en forma organizada. } \\
\text { Demuestra. Explica. Ofrece ejercitación y práctica. }\end{array}$ \\
\hline & Facilitador & Selecciona recursos. Monitorea, dirige. \\
\hline & Mediador & $\begin{array}{l}\text { Recupera conocimientos previos. } \\
\text { Construye andamiaje. Ofrece retroalimentación. }\end{array}$ \\
\hline Rol del estudiante & Receptivo & $\begin{array}{l}\text { Percibe los vínculos entre las ideas. } \\
\text { Usa herramientas de mediación propuestas. Toma apuntes. } \\
\text { Responde a la solicitud de seguimiento metacognitivo. }\end{array}$ \\
\hline
\end{tabular}


Tabla 4 (continuación)

\begin{tabular}{ll}
\hline \multirow{3}{*}{ Autogestivo } & Establece su ritmo de trabajo \\
& Gestiona dispositivos de mediación \\
& Da uso particular a las mediaciones propuestas por el docente \\
& Seguimiento metacognitivo de su aprendizaje \\
\hline
\end{tabular}

Fuente: Elaboración propia

Resultado: El fin último que motiva el sistema de actividad educativo es el logro de aprendizajes como construcción activa inseparable de la acción y reflexión (Russell, 1997; Juárez, et al, 2008; Havnes, 2004; Pham, 2016; Mattar, 2018). En la República Argentina, la educación universitaria de calidad se orienta a la formación de un profesional competente capaz de actuar con solidez profesional, responsabilidad, espíritu crítico y reflexivo, mentalidad creadora, sentido ético y sensibilidad social, resultado de las acciones de distintas instancias de participación en la universidad (Ley de Educación Superior, art 28, inciso a).

En las investigaciones analizadas, no se establecen categorías de análisis para la historicidad y multivocalidad. En cuanto a la presencia de contradicciones, asumen la clasificación propuesta por Engeström (1987), descritas en la tabla 5.

Tabla 5: Contradicciones del sistema de actividad

\begin{tabular}{ll}
\hline Primario & En un nodo del sistema de actividad \\
\hline Secundario & Entre dos o más nodos del sistema de actividad \\
\hline Terciario & Cuando un objeto más desarrollado entra en el sistema de actividad \\
\hline Cuaternario & $\begin{array}{l}\text { Ocurre entre la actividad central y las actividades vecinas, desencadenado por la } \\
\text { contradicción terciaria. }\end{array}$ \\
\hline
\end{tabular}

Fuente: Engeström (1987)

\section{Construcción del método de análisis de las buenas prácticas desde el lente del sistema de actividad.}

Las categorías descritas en el apartado anterior, si bien resultan significativas para analizar la práctica educativa desde su configuración como sistema de actividad, no consideran aspectos aportados por el análisis desde las categorías didácticas (Sacristán y Pérez, 1989; De Vincenzi, 2009). En consecuencia, se elaboró la matriz de análisis de la práctica educativa que integra ambos marcos teóricos, teoría de la actividad y dimensiones didácticas. Se decidió utilizar el formato propuesto por Engeström (2001) para la construcción de matrices de análisis del aprendizaje expansivo, compuesto por preguntas para los elementos que configuran el sistema de actividad.

Un aspecto importante tenía que ver con la evolución de la práctica a lo largo del tiempo, producto del trabajo reflexivo del docente (principio de historicidad), para lo cual se incluyeron preguntas alusivas a la construcción del objeto de aprendizaje, los artefactos de mediación, integración de comunidades con docentes de otras cátedras y participación de egresados, normas y distribución del trabajo. Además, se incluyeron dos preguntas generales: ¿Considera que los procesos de acreditación de la carrera coordinados por la CONEAU han incidido en la mejora de 
su práctica docente?, ¿Qué cambios se advierten en el ejercicio de la práctica? Para relevar las contradicciones se siguen las cuatro categorías descritas por Engeström (2001).

Para el análisis de la multivocalidad se consideró la recopilación de información desde diversas instancias: observación de clases, entrevista semiestructurada los docentes y focus group con estudiantes. Cada curso fue observado por las integrantes del equipo de investigación en tres oportunidades. Al cierre de cada observación, se realizaron entrevistas con el profesor titular para recabar su percepción sobre la práctica educativa transcurrida. Promediando el semestre, se realizó una entrevista en profundidad con cada profesor titular y al cierre del curso se realizaron encuentros grupales con cinco estudiantes voluntarios de cada curso.

Tal como se describe en la sección Método del presente artículo, el procedimiento contempló la elaboración de preguntas vinculando cada una de las categorías didácticas, registradas en el vértice superior del gráfico de la segunda generación, con las categorías que configuran el sistema de actividad. Desde la perspectiva ecología, el aula de clases, como espacio físico y psicosocial, configura un marco de convivencia singular donde convergen diversas trayectorias sociales, históricas y culturales; estructura y organiza actividades, roles y normas de la actividad educativa. Constituye un contexto rico en interacciones, que requiere de marcos metodológicos y categorías teóricas como herramientas para su lectura y explicación, interpelando su singularidad y natural ordenamiento, sin cristalizarse en explicaciones totalizantes (Daraio y Muller, 2019). El método de investigación de las prácticas educativas representa los movimientos de lo simple a lo complejo y de lo abstracto a lo concreto que se suceden en el espacio de creación de aprendizajes (Solórzano y García, 2016).

En la figura 2, a modo de ejemplo, se muestran las preguntas formuladas para el análisis de la categoría planificación como artefacto mediador dentro del sistema de actividad y su representación gráfica considerando la segunda generación de la teoría de la actividad.

Seguidamente, se recogen las preguntas formuladas para el análisis de la práctica educativa desde las categorías de la configuración del sistema de actividad en interrelación con las categorías didácticas (tabla 6). Las preguntas formuladas se utilizaron para la elaboración de las guías de observación de clases, entrevistas a los docentes y entrevista grupal a los estudiantes. 
Figura 2: Preguntas para el análisis de la categoría planificación como artefacto mediador

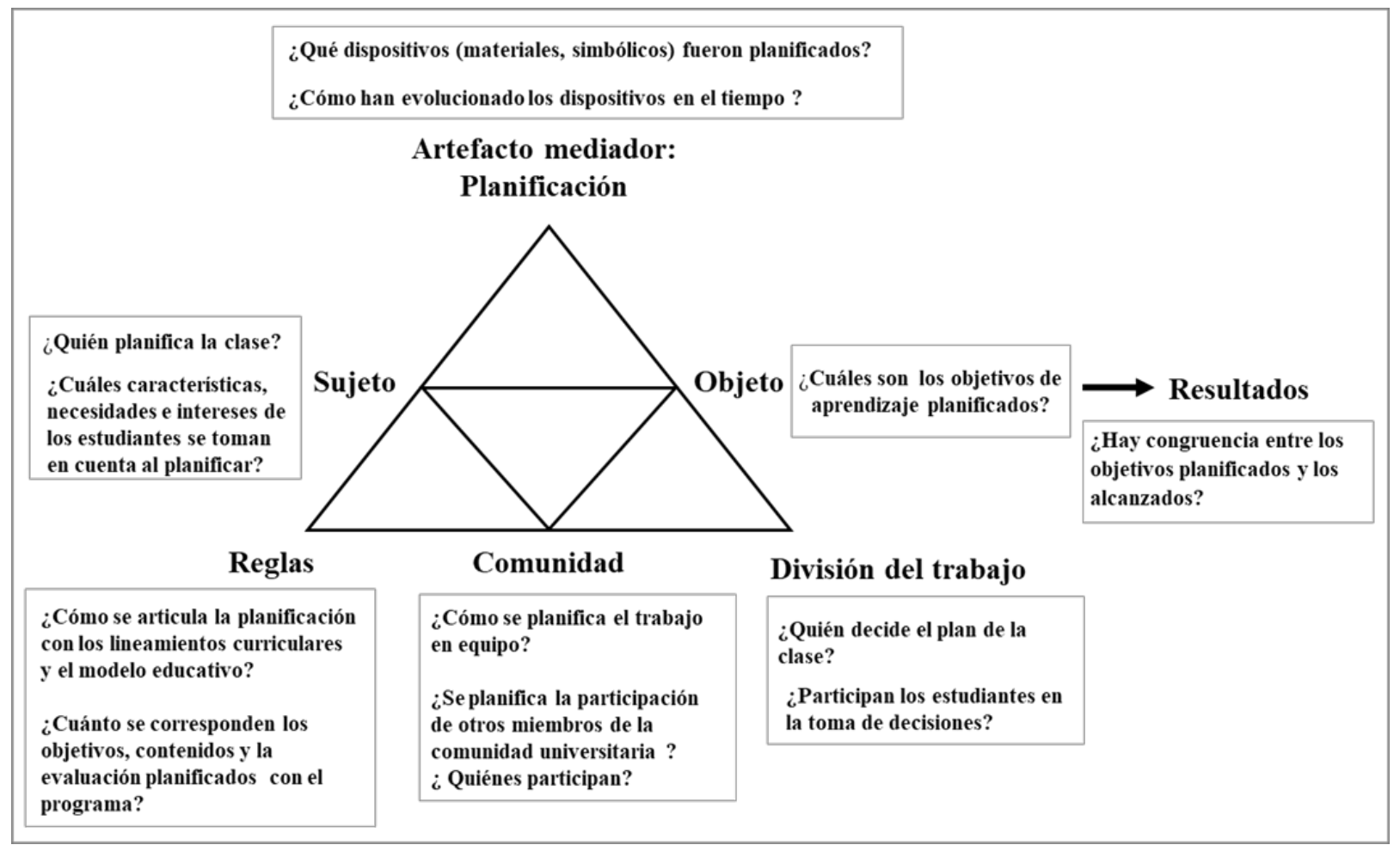

Fuente: Elaboración propia

Tabla 6: Preguntas para el análisis de las categorías del sistema de actividad

\begin{tabular}{|c|c|c|}
\hline \multirow{6}{*}{ Sujeto } & Planificación & $\begin{array}{l}\text { ¿Quién planifica la clase? ¿Cuáles características, necesidades e intereses } \\
\text { de los estudiantes se toman en cuenta al planificar? }\end{array}$ \\
\hline & $\begin{array}{l}\text { Estructura } \\
\text { metodológica del } \\
\text { contenido }\end{array}$ & $\begin{array}{l}\text { ¿Quién presenta los contenidos? ¿Cómo se distribuye el flujo de los } \\
\text { contenidos entre docentes y estudiantes? }\end{array}$ \\
\hline & $\begin{array}{l}\text { Interacción docente - } \\
\text { alumnos }\end{array}$ & ¿Quiénes interactúan y cómo lo hacen? \\
\hline & Evaluación & $\begin{array}{l}\text { ¿Quién evalúa? ¿Quién monitorea el desempeño de los estudiantes? } \\
\text { ¿Quiénrealiza las retroalimentaciones (devolución)? }\end{array}$ \\
\hline & $\begin{array}{l}\text { Organización de la } \\
\text { vidaen el aula }\end{array}$ & $\begin{array}{l}\text { ¿Quiénes participan en la organización del grupo clase, espacio, tiempo y } \\
\text { recursos? }\end{array}$ \\
\hline & Tareas académicas & ¿Quiénes participan en las tareas? \\
\hline \multirow{4}{*}{$\begin{array}{l}\text { Artefacto de } \\
\text { mediación }\end{array}$} & Planificación & $\begin{array}{l}\text { ¿Qué dispositivos (materiales, simbólicos) fueron planificados? } \\
\text { ¿Cómo han evolucionado los dispositivos durante su práctica? }\end{array}$ \\
\hline & $\begin{array}{l}\text { Estructura } \\
\text { metodológicadel } \\
\text { contenido }\end{array}$ & $\begin{array}{l}\text { ¿Cómo se estructuran metodológicamente los contenidos en clase? } \\
¿ \text { ¿Se revisan los conocimientos y creencias previas? }\end{array}$ \\
\hline & $\begin{array}{l}\text { Interacción docente- } \\
\text { alumnos }\end{array}$ & ¿Cómo se evalúa la participación de los estudiantes? \\
\hline & Evaluación & $\begin{array}{l}\text { ¿Cómo se monitorea el desempeño de los estudiantes? ¿Cómo se realizan } \\
\text { las devoluciones de resultados (retroalimentación)? ¿Qué instrumentos } \\
\text { de evaluación se utilizan en clase? }\end{array}$ \\
\hline
\end{tabular}




\section{La práctica educativa bajo el lente de la teoría de la actividad}

Tabla 6 (continuación)

\begin{tabular}{|c|c|c|}
\hline & $\begin{array}{l}\text { Organización de la vida } \\
\text { en el aula }\end{array}$ & ¿Cómo se organiza el espacio y el tiempo del aula? \\
\hline & Tareas académicas & $\begin{array}{l}\text { ¿Qué recursos se disponen para la realización de las tareas y con qué } \\
\text { finalidad? ¿Quién propone los artefactos y recursos para las tareas? }\end{array}$ \\
\hline \multirow{6}{*}{ Objeto } & Planificación & ¿Cuáles son los objetivos de aprendizaje planificados? \\
\hline & $\begin{array}{l}\text { Estructura metodológica } \\
\text { del contenido }\end{array}$ & $\begin{array}{l}\text { ¿Cómo se estructuran los contenidos en torno de los objetivos de } \\
\text { aprendizaje? }\end{array}$ \\
\hline & $\begin{array}{l}\text { Interacción docente - } \\
\text { alumnos }\end{array}$ & $\begin{array}{l}\text { ¿La interacción docente-alumnos se desarrolla en torno al objetivo? } \\
\text { ¿Cómo lo hacen? }\end{array}$ \\
\hline & Evaluación & $\begin{array}{l}\text { ¿Se corresponden los procedimientos de evaluación con los objetivos de } \\
\text { aprendizaje? }\end{array}$ \\
\hline & $\begin{array}{l}\text { Organización de la vida } \\
\text { en el aula }\end{array}$ & $\begin{array}{l}\text { ¿De qué manera la organización de la clase influye en el logro de los } \\
\text { objetivos? }\end{array}$ \\
\hline & Tareas académicas & ¿Qué relación guarda la tarea académica con el objetivo de aprendizaje? \\
\hline \multirow{6}{*}{ Reglas } & Planificación & $\begin{array}{l}\text { ¿Cómo se articula la planificación con los lineamientos curriculares y el } \\
\text { modelo educativo? ¿Cuánto se corresponden los objetivos, contenidos y la } \\
\text { evaluación planificados con el programa? }\end{array}$ \\
\hline & $\begin{array}{l}\text { Estructura metodológica } \\
\text { del contenido }\end{array}$ & $\begin{array}{l}\text { ¿Qué criterios se utilizan para la selección y secuenciación de los } \\
\text { contenidos? ¿De qué forma se relacionan los contenidos con las } \\
\text { prescripciones disciplinares y legales de la práctica profesional? }\end{array}$ \\
\hline & $\begin{array}{l}\text { Interacción docente - } \\
\text { alumnos }\end{array}$ & $\begin{array}{l}\text { ¿Qué consignas de trabajo se utilizan para promover la interacción? ¿Los } \\
\text { acuerdos de interacción son implícitos o explícitos? }\end{array}$ \\
\hline & Evaluación & ¿Qué criterios evalúan el logro de los objetivos de aprendizaje? \\
\hline & $\begin{array}{l}\text { Organización de la vida } \\
\text { en el aula }\end{array}$ & $\begin{array}{l}\text { ¿Cuál es el uso permitido del tiempo, espacio y recursos en el desarrollo } \\
\text { dela clase? ¿Cómo se estructuran las tareas? }\end{array}$ \\
\hline & Tareas académicas & ¿Cómo son las consignas que orientan la tarea a realizar? \\
\hline \multirow{6}{*}{ Comunidad } & Planificación & $\begin{array}{l}\text { ¿Cómo se planifica el trabajo en equipo? ¿Se planifica la participación de } \\
\text { otros miembros de la comunidad universitaria? ¿Quiénes participan? }\end{array}$ \\
\hline & $\begin{array}{l}\text { Estructura metodológica } \\
\text { del contenido }\end{array}$ & $\begin{array}{l}\text { ¿Se estructuran los contenidos de la clase con la participación de todos? } \\
\text { ¿Cómo se construyen? ¿Quiénes participan? ¿Cómo participa la cátedra? }\end{array}$ \\
\hline & $\begin{array}{l}\text { Interacción docente - } \\
\text { alumnos }\end{array}$ & $\begin{array}{l}\text { ¿Cómo se describe el clima de trabajo? ¿Cómo se promueve la } \\
\text { cooperaciónentre pares y el debate crítico? }\end{array}$ \\
\hline & Evaluación & $\begin{array}{l}\text { ¿Cómo participa el grupo clase en la evaluación? ¿Cómo participa la } \\
\text { cátedray la facultad en la evaluación? ¿Participan miembros de la } \\
\text { comunidad profesional? }\end{array}$ \\
\hline & $\begin{array}{l}\text { Organización de la vida } \\
\text { en el aula }\end{array}$ & $\begin{array}{l}\text { ¿Se favorece el trabajo colaborativo y la conformación de comunidad de } \\
\text { aprendizaje? ¿Quiénes integran la comunidad de aprendizaje? ¿Qué } \\
\text { elementos son importantes? }\end{array}$ \\
\hline & Tareas académicas & $\begin{array}{l}\text { ¿Cómo se relacionan las tareas con la comunidad intra y extra } \\
\text { universitaria? }\end{array}$ \\
\hline \multirow{3}{*}{$\begin{array}{l}\text { División del } \\
\text { trabajo }\end{array}$} & Planificación & $\begin{array}{l}\text { ¿Quién decide el plan de la clase? ¿Participan los estudiantes en } \\
\text { la tomade decisiones? }\end{array}$ \\
\hline & $\begin{array}{l}\text { Estructura metodológica } \\
\text { del contenido }\end{array}$ & ¿Quién selecciona y establece la secuencia de los contenidos? \\
\hline & $\begin{array}{l}\text { Interacción docente - } \\
\text { alumnos }\end{array}$ & $\begin{array}{l}\text { ¿Cómo interaccionan los estudiantes entre sí? ¿Interactúan con el } \\
\text { contexto? ¿Quién y cómo se promueve? ¿Qué tipo de interacciones } \\
\text { docente alumnos predominan? }\end{array}$ \\
\hline
\end{tabular}


Tabla 6 (continuación)

\begin{tabular}{|c|c|c|}
\hline & Evaluación & $\begin{array}{l}\text { ¿Se proponen instancias para la autoevaluación y la coevaluación? ¿Cómo } \\
\text { lo hacen? }\end{array}$ \\
\hline & $\begin{array}{l}\text { Organización de la vida } \\
\text { en el aula }\end{array}$ & ¿Cómo incide la distribución de los roles en el clima de la clase? \\
\hline & Tareas académicas & $\begin{array}{l}\text { ¿Cómo se pauta la distribución de roles en la ejecución de las tareas } \\
\text { académicas? }\end{array}$ \\
\hline \multirow{6}{*}{ Resultados } & Planificación & ¿Hay congruencia entre los objetivos planificados y los alcanzados? \\
\hline & $\begin{array}{l}\text { Estructura metodológica } \\
\text { del contenido }\end{array}$ & $\begin{array}{l}\text { ¿Los resultados responden a la selección y estructuración de los } \\
\text { contenidos? }\end{array}$ \\
\hline & $\begin{array}{l}\text { Interacción docente - } \\
\text { alumnos }\end{array}$ & $\begin{array}{l}\text { ¿Los resultados son producto de la interacción entre pares y con el } \\
\text { docente? }\end{array}$ \\
\hline & Evaluación & $\begin{array}{l}\text { ¿Qué habilidades, destrezas y actitudes aprenden los estudiantes? ¿Qué } \\
\text { evidencias se utilizan para evaluar los resultados? }\end{array}$ \\
\hline & $\begin{array}{l}\text { Organización de la vida } \\
\text { en el aula }\end{array}$ & ¿Cómo se organiza la clase en torno a los resultados esperados? \\
\hline & Tareas académicas & ¿Qué resultados se alcanzan con las tareas prescriptas? \\
\hline
\end{tabular}

Fuente: Elaboración propia

\section{Conclusiones}

Del proceso investigativo, sus resultados y discusión, se concluye que:

1. El método propuesto para la investigación de buenas prácticas educativas resultó lo suficientemente amplio para dar cabida a una variedad de voces y niveles de análisis, y adecuadamente profundo al representar de manera gráfica la complejidad de interacciones socioculturales responsables del aprendizaje. La batería de preguntas interpeló la experiencia áulica, el proceso de construcción de un objeto de aprendizaje iniciado por motivaciones individuales y transformado en objeto colectivo de aprendizaje, mediado por una variedad de artefactos materiales y simbólicos. De la misma manera, las categorías y preguntas permitieron transcender la observación parcelada del aula, ofreciendo un lente integrador del triángulo sujeto - herramientas - objeto, con factores sociales interconectados en comunidad de aprendizaje, con arreglo de roles y restricciones que garantizan la práctica educativa de calidad. Las categorías de análisis de la teoría de la actividad, configuración de los sistemas de actividad, historicidad y contradicciones permiten analizar la complejidad dinámica de la práctica áulica con participación de los distintos actores y su evolución histórica y cultural.

2. Las categorías y preguntas del método elaborado abonan a la construcción de esquemas de investigación para el estudio en profundidad de las buenas prácticas en el contexto del aula, dando cuenta de las reorganizaciones y transiciones con participación de distintos actores en la evolución histórica sociocultural de la enseñanza como práctica altamente institucionalizada.

3. Cruzar las categorías del sistema de actividad con las dimensiones de la práctica docente desde la didáctica, posibilitó la construcción de un lente capaz de captar la complejidad de una práctica educativa, así como también constituyó el insumo para la elaboración de los instrumentos de recolección de información. 


\section{La práctica educativa bajo el lente de la teoría de la actividad}

\section{Referencias}

Arnseth, H. (2008). Activity theory and situated learning theory: contrasting views of educational practice. Pedagogy, Culture \& Society, 16(3), 289-302. https://doi.org/10.1080/14681360802346663

Christiansen, E. (1996). Tamed by a rose: Computers as tools in human activity. En B. A. Nardi (Ed.), Context and consciousness: Activity theory and human-computer interaction, (pp. 175-198). Cambridge, MA: MIT Press https://doi.org/10.1109/tpc.1997.649561

Comisión Nacional de Evaluación y Acreditación Universitaria. (2020, Octubre 12). https://www.coneau.gob.ar/coneau/que-es-la-coneau/

Daraio, V. y Müller, M. (2019). Formación docente: dar clases con donación. Educación, Lenguaje y Sociedad, 17(17), 1-17. https://cerac.unlpam.edu.ar/index.php/els/article/view/4211

Daniels, H. (2016). Uma análise da teoria da atividade de aprendizagem no e para o trabalho interescolar. Educação, 39(4), 24-31. https://revistaseletronicas.pucrs.br/ojs/index.php/faced/article/view/24319

De Vincenzi, A. (2009). Concepciones de enseñanza y su relación con las prácticas docentes: un estudio con profesores universitarios. Educación y Educadores, 12(2), 87-101.

http://www.scielo.org.co/scielo.php?script=sci_arttext\&pid=S0123-12942009000200007\&lng=en\&nrm=iso\&tlng=es

Edenstein, G. (2000). El análisis didáctico de las prácticas de la enseñanza. Una referencia disciplinar para la reflexión crítica sobre el trabajo docente. Revista del Instituto de Investigaciones en Ciencias de la Educación. $17,3-7$.

Engeström, Y. (1987). Learning by expanding: An activity-theoretical approach. Helsinki: Orienta Konsultit. https://www.tandfonline.com/doi/abs/10.1080/13639080020028747

Engeström, Y. (2001). Expansive Learning at Work: Toward an activity theoretical reconceptualization. Journal of Education and Work, 14(1), 133-156. https://doi.org/10.1080/13639080020028747

Engeström, Y. (2014) Activity theory and learning at work. In Deinet, U, \& Reutlinger, C. (Eds). TätigkeitAneignung-Bildung: Positionierungen zwischen Virtualität und Gegenständlichkeit (pp. 67-96). Springer-Verlag. https://doi.org/10.1007/978-3-658-02120-7_3

Foot, K. A. (2014). Cultural-historical activity theory: Exploring a theory to inform practice and research. Journal of Human Behavior in the Social Environment, 24(3), 329-347. https://doi.org/10.1080/10911359.2013.831011

Gimeno, J. y Pérez, A. (1989). La enseñanza: su teoría y su práctica. Madrid: Akal Universitaria.

Gimeno, J. y Pérez, A. (1992). Comprender y transformar la enseñanza. Madrid: Ediciones Morata.

Grupo DIM - Universidad Autónoma de Barcelona (2014). Grupo DIM-Didáctica, Innovación, Multimedia. Barcelona: Grupo DIM

http://dim.pangea.org/dimnewinvestigaciones.htm

IPSA SCIENTIA - Vol. 5 Nº $1-2020$ 


\section{La práctica educativa bajo el lente de la teoría de la actividad}

Hardman, J. (2008). Researching pedagogy: An activity theory approach. Journal of Education, 45(1), 65-95. https://bit.ly/344djHr

Harvey, L., \& Green, D. (1993). Defining quality. Assessment \& evaluation in higher education, 18(1), 9-34. https://doi.org/10.1080/0260293930180102

Havnes, A. (2004). Examination and learning: an activity-theorical analysis of the relationship between assessment and educational practice. Assessment \& Evaluation in Higher Education, 29(2), 159-176. https://doi.org/10.1080/0260293042000188456

Huang, W. (2011). Learning in authentic contexts: Projects integrating spatial technologies and fieldwork. Journal of Geography in Higher Education, 35 (4), 565-578. https://doi.org/10.1080/03098265.2011.559577

Jonassen, D. (2000). Revisiting activity theory as a framework for designing student-centered learning environments. In Jonassen, D. \& Land, S. (Eds), Theoretical foundations of learning environments (89-122) Lawrence Erlbaum Associates, Inc. https://epk.is/F0Y9h

Juárez, M. Buenfil, R. y Triguelos, M. (2008). De las prácticas convencionales a los ambientes de aprendizaje colaborativo a distancia. Un estudio con profesores de ciencias de bachillerato desde la teoría de la actividad. Revista Mexicana de Investigación Educativa, 1005-1083. http://www.scielo.org.mx/scielo.php?script=sci_arttext\&pid=S1405-66662008000400003

Leontiev, A. N. (1981). Problems of the development of the mind. Moscow: Progress Publishers.

Kumpulainen \& Sefton-Green, (2014). What is connected learning and how to research it? International Journal of Learning and Media April, 4(2). Massachusetts Institute of Technology. https://doi.org/10.1162/IJLM_a_00091

Ley 2452. Ley de Educación Superior. Art. 8, inciso a. Publicada el 20 de julio de1995. Argentina.

Lucarelli, E. (2006). Análisis de prácticas y formación: tres experiencias con docentes en universidades argentinas. Perspectiva, 24(1), 273-296.

Mattar, J. (2018). Constructivism and connectivism in education technology: Active, situated, authentic, experiential, and anchored learning. Revista Iberoamericana de Educación a Distancia, 21(2), 201-217. https://doi.org/10.5944/ried.21.2.20055

Nussbaumer, D. (2012). An overview of cultural historical activity theory (CHAT) use in classroom research 2000 to 2009. Educational review, 64(1), 37-55. https://doi.org/10.1080\%2F00131911.2011.553947

Perkins, D. (2001). La escuela Inteligente. Del adiestramiento de la memoria a la educación de la mente. Barcelona: Gedisa.

Pham, T. (2016). Student-centredness: Exploring the culturally appropriate pedagogical space in Vietnamese higher education classrooms using activity theory. Australian Journal of Teacher Education (Online), 41(1), 1-21. https://doi.org/10.14221/ajte.2016v41n1.1

Salas-Madriz, F. (2016). Aportes del modelo de Yrjö Engeström al desarrollo teórico de la docencia universitaria. Revista Educación, 40(2), 1-22. https://doi.org/10.15517/revedu.v40i2.15257 


\section{La práctica educativa bajo el lente de la teoría de la actividad}

Salomon, G. (1993). No hay distribución sin la cognición de los individuos: un enfoque interactivo dinámico. En G. Salomon (comp.). Cogniciones Distribuidas. Consideraciones Psicológicas y Educativas (pp. 153-184). Buenos Aires: Amorrortu.

Sannino, A., \& Engeström, Y. (2018). Cultural-historical activity theory: founding insights and new challenges. Cultural-Historical Psychology, 14(3), 43-56.

https://doi.org/10.17759/chp.2018140304

Solórzano, F y García, A. (2016). Fundamentos del aprendizaje en red desde el conectivismo y la teoría de la actividad. Revista Cubana de Educación Superior, 35(3), 98-112. http://scielo.sld.cu/scielo.php?pid=S0257-43142016000300008\&script=sci_arttext\&tlng=pt

Soloviova, Y. (2019). Las aportaciones de la teoría de la actividad para la enseñanza. Educando para educar, (37), 13-24. https://dialnet.unirioja.es/servlet/articulo?codigo=7186597

Russell, D. (2004). Activity theory and distributed learning. The Routledge Falmer reader in psychology of education, 309. https://bit.ly/378Rfxm

Tena, A., y Rivas-Torres, R. (1995). Manual de investigación documental. Elaboración de tesinas. México: Plaza y Vladez, S.A.

Ventak, H. \& Adler, J. (2008). Expanding the foci of activity theory: accessing the broader contexts and experiences of mathematics education reform. Educational Review, 60(2), 127-140. https://doi.org/10.1080/00131910801933914

Vygotsky, L. S. (1978). Mind in society: The development of higher psychological processes. Cambridge: Harvard University Press.

Wenger, E. (2010). Communities of Practice and Social Learning Systems: the Career of a Concept. Social Learning Systems and Communities of Practice, 179-198. https://doi.org/10.1007/978-1-84996-133-2_11

Zabalza, M. (2012). El estudio de las "buenas prácticas” docentes en la enseñanza universitaria. REDU. Revista de Docencia Universitaria. Monográfico: Buenas prácticas docentes en la enseñanza universitaria, 10(1), 17-42. https://doi.org/10.4995/redu.2012.6120 